\title{
HEMATOMA SUBDURAL Y FRACTURA DE LOS HUESOS LARGOS
}

\author{
Relato de un caso.
}

\author{
Por los Dess, J. Meneghello y J. HASBun (*) \\ Hoop. M. Atriarán, Cátedras de Padiatria de los Profs. A. Bzeza Goñi y J. Meneghe:lo Rivera.
}

No más cte una docena de casos se han putlicado hasila la fecbia acerca de esta curiosa asociación de hematoma subdural y fractura de los huesos largos. El primero que llamó la atención a este hecho fué Caffey', quien, en 1946, publica un trabajo con un relato de seis obsorvaciones de lactantes con fracturas de los huesos largos como complicación del hematoma cubilural. Aunque en todos sus casos las fracturas parecian ser de origen traumátioo, no se pudo obtener on ringuno de ellos po: la historia, el antecedente priciso del tranmatisno cansal. La mayoría de las fracturas aparecieron después del comienzo do los hematomas cubdturales y no so encontró en ningún caro evidencia, ni clínica ni radiológica, de alguna enfermedad pre-existente dol sistema esquelético que pudiera predisponer a las fracturas patológicas. Concluye que las fracturas de los buesos largos son una complicación común del hematoma subdural infantil y recomienda que cuando se esté en presencia de un lactante con fracturas inexplicables de liss huesos largos, hay que orientar la investigación hacia el hematoma subdural. Además, sugiere que en todo caso de hematonia subetural se practique un exam?n rađiológico do rutina de los huesos largos, ya que muchas fracturas son clínicamente silenciosas.

Smith $^{2}$, en 1950, relata un nuevo caso cn un lactante de $4 \%$ meses, con múltiples fracturas de los huesos largos y del cráneo, sin antecedentes traumáticos y al

(*) Servicio de Radiologia del Hospital Mantel Arriarán, cual se le hizo el diagnóstico de hematoma subdural por este solo hallazgo, ya que la historia y cxamen físico no suministraban clementos para pensar en él. No se comprobó ninguna enfermedad capaz de producir fracturas patológicas. A fines de 1950 , Lis y Frauenberger ${ }^{3}$ publican otro caso, en un lactante de 10 meses de edad. con fracturas múltiples costales y del miembro inferior, sin que se pudiera comprobar antecedente traumático y en el que por la clínica se sospechó el hematoma strbdural, diagnóstico que fué confirmado en la intervención.

La observación clínica que presenta mos a continuación ilustra acerca de esta combinación con semejanzas llamativas a los cinos relatados en la literatura.

M. S. V. - Obs. $N^{5} 75647 / 50$ - Lactante de sexo femenino, de 1 año de edad, cuyos antecedentes personales no fué posible precisar, porque su madre falleció 2 meses antes a consecuencla de un aborto y la persona a cargo de ella sólo precisa de que siempre fué vomitadota y que sufria de estreñfmlento. Además, fué muy retrasada en su desarrollo estấtico-dinámico y muy irritable. El día $10 \mathrm{de}$ octuire de 1950 es hospitalizeda en la Posta Infantil, porque desde 15 dias antes tiene decaimiento y reacción febrll; en los últimos o días se había agravado, estaba somnolienta, presentaba vérnitos y constipación. Vista por médico particular prescribe antiblóticos y fenobarbital en dosls elevadas. En recepclón se encontró una enfermita inconsciente, con pésimo estado nutritivo, deshidratada, que no reaccionaba al examen, con enfriamiento e htpotcinía generalfzada, miosis pupilar bilatoral, resplración superficial y lenta y equimosis facial bilateral. Se plantearon los diagnósticcs 
de meningoencefajitis o intoxicación barbltúrica, dado el antecedente de la administración de fenobarbital en dosis altas $(0.10 \mathrm{~g}$ 2 veces al dial. Se instituyó como tratamiento de urgencia, estricnina, oxígeno, calen $* a-$ mlento e hidratación paraenteral. Al dia suisigulente está consciente y es dada de alta 6 clias despues, en mejores condiciones, afebril $y$ alimentándose en buena forma, con la indicación de control en la Policlinica.

Cuatro días más tarde consulta en la Policlínrca por presentar transitoriamente deposiciones mucosas y vómitos repetidos. Por el mal estado nutritivo y la deshidratación se decide hospitalizarla nuevamente, ahora en la secclín A del Hospital M. Arriarán, el día 25-X-50. Fri el servicio se comprueba un lactante con deficiente estado general y nutritivo, con un peso de $5,880 \mathrm{~g}$ y una talla de $70.5 \mathrm{~cm}$. Fstá consciente e imitable cuando se trata de examinarla; yace en decúbito dorsal $y$ con las extremidades en abducctón y flexión. Hay deshidrataión de la piel e hiperpigmentación del dorso de las manos, pies y cara externa de los antebrazos, además, numerosas zonas equimóticas, que comprometen especialmente la region malar e infraocular izquierda. Hay, además, tinte violáceo de la cara externa de los tobillos y dorso de los pies. El cráneo es asimétrico $y$ ensanchado en el diámetro biparietal. Las encías están hiperémlcas, pero no sangran. La Iengua está húmeda y rosada. El tórax se aprecia enflaquecido y prominente en quilla. El examen pulmonar y cardiaco no revela anormalidades. El abdomen se presenta excarado y no se palpan visceras. Las extremidades inferiores se ven en actitud pasiva, inmovilizadas voluntariamente en flexión, abducción y rotación externa del muslo; la movilización provocada por el examen le causa dolor. En el tercio inferior de ambas piernas se observa una deformación lateral externa, dolorosa y con movilidad anormal elástica. Ia tonicidaci está conservada. La impresión diagnóstia en el servicio es de distrofia grave, sindrome purpurico, zescorbuto? y fractura de las extremidades inferiores.

Se practicaron los siguientes exámenes die laboratorio: el hemograma revela: $4.240,070$ eritrocitos con $73,9 \%$ de $H$ b, 5,600 leucoeitos con $4 \%$ de bacihlformes, $58 \%$ de segmentados, $16 \%$ de linfositos, $12 \%$ de monocitos y $9 \%$ de eosinófilos. Plaquetas normales al frotis. El tiempo de coagulación es de 2 minutos $y$ el de sangria es de 1 minuto. Test de Rumpel Leede negativo. El nivel de vitamina $C$ en ayunas reveló cifras iniciales bajas (0.480 $\mathrm{mg}$ cr. ?. Los exámenes de orina y urocultivos negativos.

E1 estudio radiográfico de las extremidades inferiores informó lo slgulente: fractura bilateral simétrica $y$ completa a nivel de la metáfisis distal de los huesos de la plerna; los fragmentos en buena posicion. No hay signos evidentes de escorbuto.

En vista de estos hallazgos se conversj nuevamente con el padre y con su cuidadora, obteníndose sólo datos vagos e imprecisos respecto a traumatismos que pudieran ser los causantes de las fracturas.

Su evolución en el servicio: los primeros 15 dias llama la atención por su mal estado general, el decaimiento, sus frecuentes vomitos, la marcada hidrolabilidad, que abligan a

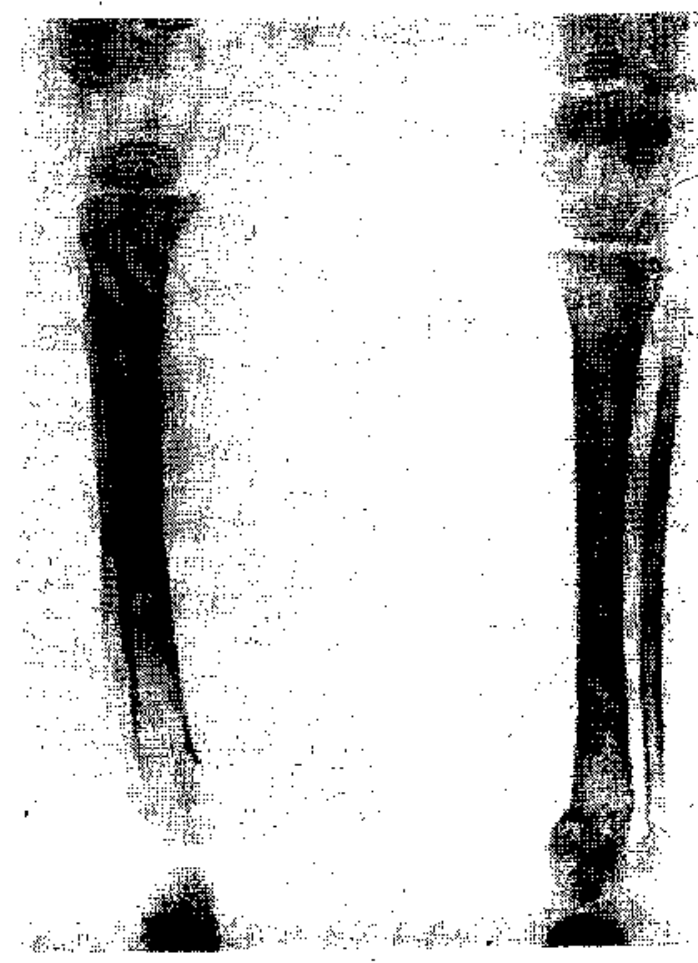

Radiografía de ambas pieroas: fractura bilateral. simetrica, a nivel de la metáfisis distal de los buesos de la pietna.

hidratación paraenteral gota a sota y plasmas; ol estacionamiento en el peso, su dificultad para alimentarse y un estreñimiento pertimaz. El sindrome purpúrico desaparece en un lapso aproximado de 2 semanas. A partir de la segunda semana está subjetivamente mejor, más hidratada, pero persisten los vómitos y la constipación. Delbido a la rebeldía de estos sintomas se practica un estudio radiológico del tubo algestivo y cuyo informe dice: estómago grande, hipotónico. Buen pasaje pilórtco: duodeno normal. Una hora después ha pasado sólo pequeña cantidad de bario a las asas delgadas. Seis horas más tarde: restos de la papilla opaca en el estómago; el bario contrasta las últimas porciones del ileum y el colon hasta el ángulo hepático. A 
las 54 horas está inyectado el colon ascandente, transverso y descendente, los que se observan de aspecto normal; a las 96 horas hay barlo en el calon sigmoideo y ampolla rectal. Conclusión radiológica: atonia gástrica; constipación colónica. También se practlcó un enema baritado, el que reveló un colon de tamaño $\mathrm{y}$ forma normales $\mathrm{y}$, además, una disquexia rectal.

Vistos los resultados del examen radiológico del tubo digestivo, se ensaya tratamiento a base de vitamina $\mathbf{B} 1$ y prostigmlna, con 10 que se logra normalizar el tránsito intestinaj.

En conocimiento del trabajo publicado por Caffey y dada la similitud de nuestra enfermita con las observaciones publicadas por él, pensamos en el hematoma subdural, diagnóstico que se hizo más evidente cuando alginos días después se advierte un estrabisino convergente bilateral y una parálisis facial derecha.

El dia 29-XI-50 se practica una punsion subdural: en el lado izquierdo sale liquido teniido de sangre en un comienzo y luego intensamente xantocrómico. Se dejan escapar más o menos $10 \mathrm{cc}$; en las últimas porclones Plielve a teñjrse de sangre. A derecha la punclón dío salida a liquido hemorrágico. que fluyó gota a gota rápida y no cambió de co. loración hasta el fínal. Se dejan flulr $10 \mathrm{cc}$. Las resultados de sus exámenes químico y citológico son los sizulentes: liquido subdural derecho, intensamente hemorrágico; Pandy, ++++ ; leucocitos, $10 \times \mathrm{mm}$. El liquido subdural izquierdo es hemorrágico, con Pandy, ++++ ; albimina, $1.80 \mathrm{~g}$; glucosa, $0.50 \mathrm{~g}$; coloruros, $7,2 \%$; leucocitos, $4 \times \mathrm{mm}$; cultivo $(-)$.

Por esta época se practica un fondo de ojo que revela edema papiar bilateral y un examen neurolósico en que sólo llama la atención una tonicidad muscular disminuida y los reflejos tendinosos vivos en ambas extremidades. Se sienta con dificultad.

Un examen de fosfemia revela: $4.3 \mathrm{mg} \%$ y calcemia, $11 \mathrm{mg} \%$. Calchuria: $1.80 \%$ (normal, $0.10-0.25 \%$ ). Fosfatasas: 6.5 unidades Bodansky.

Se practican 3 punciones subdurales, que dan sallda a líquido con los mismos caracteres a los ya anotados, y el día 27-XII-50 se decide practicar el segundo tiempo de la operación de Ingraham, encontrándose que a izquierda sale líquido citrino claro y se descubre una membrana firme rojiza. A derecha no se comprobó membrana de hematoma y sale líquido claro.

Es cada de alta el día 23 de enero de 1951 , después de 3 meses de hospitalización. con 2 kilos más de peso, con muy buen estado general y con la indjeación de control en la Policlinica.

\section{Comentario.}

Sucede a veces que el diagróstico do hematoma subdural en cl lactante se hace extremadamente difícil, por ausencia de los signos clásicos que fueran puntualizados an el trabajo publicado por Ingraham $^{*}$ en 1945. Es por esto que el conocimiento de esta asociación de fracturis de los huesos largos y hematoma subdural puede ser de gran utilidad al radiógo, sugiriendo al clínico esta posibilidad. cuando la sintónatología de hematoma no es claro.

Igual que en los casos relatados en la literatura no fué posible precisar en nuestro enfermo antecedentes traumáticos que explicaran las fracturas: el único hallazgo de inportancia etiológica es ol bajo nivel de vitamina $C$ encontrado an el plasma. Pero creculos que su importancia es relativa, por cuanto investigaciones practicadas por nosotros" en el año 1948, demostraron en el estudio humoral de 100 casos de niños distróficos, la existcncia do una hipoavitaminosis de cierta intensidad en todos ellos, y 1 as radiografías de los huesos largos no mostró fractura en ningún caso, aunque en dos tercios de ellos habia signos radiológicos óseos que pueden cousiderarse característicos de hipoavitaminosis C. En nuestro enfermo, fucra de la ontcopororis y el adelgazamiento ir la cortical. conuán a casi todos los estados distrófieos del niño, faltaban los otros signos clásicos del escorbuto, como la rarefacción zubmetafisiaria, el signo. de Wimberget en los núcleos de osificaciór, el engrosamiento de las líneas de calcificación provisoria, los espolones laterales y a carácter y localización mismos de las fracturas en posición más alta que lo que acontece en el escorbuto.

No sabemos cuándo aparecieron las fracturas en nuestro enfermo. Caffey señala que en 5 de 6 casos fueron posteriores al hematoma. En el caso relatado por nosotros creemos que el hematoma is intiguo, oor el ensanchamiento de la cabeza, la distrofia avanzida y al hallazso de membrana en la intervención. En 
cambio, las fracturas parecen ser recientes (ausencia de callo óseo).

Nos ha sorprendido que casi todos los casos publicados demuestran la presencia de petequias o y equimosis, cerca o lejar nos al sitio de fracturas. Como lo señlamos, nuestro pacionte lambién tenia estas manifestaciones. Ingraham, en şu vasta experiencia on hematoma subdural, no hace mención a estas manifestaciones cutáneas, pero si se refiete a las hemorragias retinales como de relativa frecuencia. Los estudios de coagulación sarguínea practicados en el caso relatado por nocotros, no mostraron anormalidad.

\section{Conclusión.}

Se presenta el caso de un lactante con fracturas de los huesos largos, ballazgo que ayudó a establecer el diagnóstico do hematoma subdural, No se precisó ia eliología. Se llama la atención a la frocuencia de manifestaciones purpúricas que acompañan a esta asociación de frac tura y hematoma subdural.
Summary.

The authors present a case of an infants with several factures of long bones associated with a subdural hematoma. The etiology was not established. They stress the frecuency of purpuric unanifestations in cases of subdural hematom:t with factures.

\section{Bibliografía.}

1.-CAFFEY, J. - Multiple Fractures in lang bones of Infants suffering from Subdural Hematoma, Am. J. Roentgenol. 56: 163, 1948.

2.-SMITH, M. J. - Subdural Hematoma with multiple Fractures. Am. J. Roentgenol. $63: 342,1950$.

3.-LIS, E. F. y FRAUIENBERGER, G. S. Mult lplefractures with Subdural Hematuma in Infancy. Pediatrics 6: $\mathbf{3 9 0}, 1950$.

4.-INGRAHAM, F. D. Y MATSON, D. D. Subdural Hematoma in Infancy. J. Pediat. 24: 1, 1945.

5.-MENEGHELLO, J.; RUBIO, S.; UNDURRAGA, O. y HASBUN, J. - Hipoavitaminosis $\mathrm{C}$ en el lactante distrófico. Rev. Chilena de Pedlatría 19: $T 2,1948$. 